\title{
Therapeutic Aspiration
}

National Cancer Institute

\section{Source}

National Cancer Institute. Therapeutic Aspiration. NCI Thesaurus. Code C65007.

The withdrawal of fluid via a needle for the purpose of treating a disease/disorder. 